\title{
A New Generalized Cassini Determinant
}

\author{
Ivica Martinjak \\ University of Zagreb, Faculty of Science \\ Bijenička 32, HR-10000 Zagreb, Croatia \\ and \\ Igor Urbiha \\ Polytechnic of Zagreb \\ Vrbik 8, HR-10000 Zagreb, Croatia
}

\begin{abstract}
In this paper we extend a notion of Cassini determinant to recently introduced hyperfibonacci sequences. We find $Q$-matrix for the $r$-th generation hyperfibonacci numbers and prove an explicit expression of the Cassini determinant for these sequences.
\end{abstract}

Keywords: hyperfibonacci numbers, Cassini identity, linear space, integer sequence, polytopic numbers

AMS Mathematical Subject Classifications: 11B39, 11B37

\section{Introduction}

Given the second order recurrence relation, defined by

$$
a_{n+2}=\alpha a_{n+1}+\beta a_{n},
$$

where $\alpha$ and $\beta$ are constants, a sequence $\left(a_{k}\right)_{k \geq 0}$ is called a solution of (1) if its terms satisfy this recurrence. The set of all solutions of (1) forms a linear space, meaning that if $\left(a_{k}\right)_{k \geq 0}$ and $\left(b_{k}\right)_{k \geq 0}$ are two solutions then $\left(a_{k}+b_{k}\right)_{k \geq 0}$ is also a solution of (11). Furthermore, it holds true that for any constant $c,\left(c a_{k}\right)_{k \geq 0}$ is also a solution of (1). Using these basic properties of a linear space one can derive the identity

$$
a_{m} b_{m-1}-a_{m-1} b_{m}=(-\beta)^{m-1}\left(a_{1} b_{0}-a_{0} b_{1}\right),
$$

where $\left(a_{k}\right)_{k \geq 0}$ and $\left(b_{k}\right)_{k \geq 0}$ are two solutions of recurrence (11) [7]. When $\alpha=\beta=1$ and initial values of the terms are 0 and 1 , respectively, the 
relation (11) defines the well known Fibonacci sequence $\left(F_{k}\right)_{k \geq 0}$. One can find more on this subject in a classic reference [8]. In case of the Fibonacci sequence relation (2) reduces to

$$
F_{n-1} F_{n+1}-F_{n}^{2}=(-1)^{n}
$$

and it is called Cassini identity [3, 6, 9]. This relation can also be written in matrix form as

$$
\operatorname{det}\left(\begin{array}{cc}
F_{n} & F_{n+1} \\
F_{n+1} & F_{n+2}
\end{array}\right)=(-1)^{n}
$$

In this paper we study the hyperfibonacci sequences which are defined by the relation

$$
F_{n}^{(r)}=\sum_{k=0}^{n} F_{k}^{(r-1)}, \quad F_{n}^{(0)}=F_{n}, \quad F_{0}^{(r)}=0, \quad F_{1}^{(r)}=1,
$$

where $r \in \mathbb{N}$ and $F_{n}$ is the $n$-th Fibonacci number. The number $F_{n}^{(r)}$ we shall call $n$-th hyperfibonacci number of $r$-th generation. These sequences are recently introduced by Dill and Mezö [2]. Several interesting theoretical number and combinatorial properties of these sequences are already proven, including those available in [1]. Here we define the matrix

$$
A_{r, n}=\left(\begin{array}{cccc}
F_{n}^{(r)} & F_{n+1}^{(r)} & \cdots & F_{n+r+1}^{(r)} \\
F_{n+1}^{(r)} & F_{n+2}^{(r)} & \cdots & F_{n+r+2}^{(r)} \\
\vdots & \vdots & \ddots & \vdots \\
F_{n+r+1}^{(r)} & F_{n+r+2}^{(r)} & \cdots & F_{n+2 r+2}^{(r)}
\end{array}\right)
$$

and we prove that $\operatorname{det}\left(A_{r, n}\right)$ is an extension of (3) . Thus, we show that the generalization of the Cassini identity, expressed in a matrix form, holds true for the hyperfinonacci sequences.

\section{$2 \quad Q$-matrix of the hyperfibonacci sequences}

According to the definition (5) obviously we have

$$
F_{n+1}^{(r)}=F_{n}^{(r)}+F_{n+1}^{(r-1)} .
$$

In case $r=1$ the second term $F_{n+1}^{(r-1)}$ is determined by the Fibonacci recurrence relation,

$$
F_{n+3}^{(1)}=F_{n+2}^{(1)}+\left(F_{n+2}^{(1)}-F_{n+1}^{(1)}\right)+\left(F_{n+1}^{(1)}-F_{n}^{(1)}\right)
$$


thus we have

$$
F_{n+3}^{(1)}=2 F_{n+2}^{(1)}-F_{n}^{(1)} .
$$

Now, iteratively using (7) we derive the recurrence relation

$$
\begin{aligned}
& F_{n+2}^{(1)}=F_{n+1}^{(1)}+F_{n}^{(1)}+1, \\
F_{n+3}^{(1)}= & F_{n+2}^{(1)}+2 F_{n+1}^{(1)}-F_{n-1}^{(1)}-F_{n}^{(1)} \\
= & F_{n+2}^{(1)}+F_{n+1}^{(1)}+2 F_{n}^{(1)}-F_{n-2}^{(1)}-F_{n-1}^{(1)}-F_{n}^{(1)} \\
= & F_{n+2}^{(1)}+F_{n+1}^{(1)}+\cdots+F_{3}^{(1)}-F_{0}^{(1)}-F_{1}^{(1)}-\cdots-F_{n}^{(1)} \\
= & F_{n+2}^{(1)}+F_{n+1}^{(1)}+1 .
\end{aligned}
$$

When $r=2$ we use the same approach to get recurrence for the second generation of the hyperfibonacci numbers,

$$
F_{n+2}^{(2)}=F_{n+1}^{(2)}+F_{n}^{(2)}+n+2 .
$$

Namely, in this case the second term in (6) is determined by obtained recurrence relation (8). This means that again we can perform the $(n+1)$-step iterative procedure, this time using

$$
F_{n+3}^{(2)}=2 F_{n+2}^{(2)}-F_{n}^{(2)}+1 .
$$

The fact that terms indexed 3 through $n$ cancel each other and that $(n+1)$ 1s remains, completes the proof of (9).

Recall that polytopic numbers are generalization of square and triangular numbers. These numbers can be represented by a regular geometrical arrangement of equally spaced points. The $n$-th regular $r$-topic number $P_{n}^{(r)}$ is equal to

$$
P_{n}^{(r)}=\left(\begin{array}{c}
n+r-1 \\
r
\end{array}\right) .
$$

When $r=3$, the $i$-th step of the iterative procedure described above results with an extra $i$, which sum to a triangular number $\left(\begin{array}{c}n+3 \\ 2\end{array}\right)$ after the final $(n+1)$ st iteration. Furthermore, in the next case we add the $i$-th triangular number in $i$-th step of iteration. According to the properties of polytopic numbers, these numbers sum to the tetrahedral number $\left(\begin{array}{c}n+4 \\ 3\end{array}\right)$. In general, in the $i$-th step of the iteration we add $i$-th regular $(r-1)$-topic number and sum of these numbers after the final step of the procedure is the regular polytopic number $\left(\begin{array}{l}n+r \\ r-1\end{array}\right)$. Now we collect all this reasoning into the following 
Lemma 1. The difference between $n$-th $r$-generation hyperfibonacci number and the sum of its two consecutive predecessors is $n$-th regular ( $r$-1)-topic number,

$$
F_{n+2}^{(r)}=F_{n+1}^{(r)}+F_{n}^{(r)}+\left(\begin{array}{c}
n+r \\
r-1
\end{array}\right) .
$$

We can also write relation (12) as

$$
F_{n+2}^{(r)}=F_{n+1}^{(r)}+F_{n}^{(r)}+P_{n+2}^{(r-1)} .
$$

Hyperfibonacci sequences can be defined by the vector recurrence relation

$$
\left(\begin{array}{c}
F_{n+1}^{(r)} \\
F_{n+2}^{(r)} \\
\vdots \\
F_{n+r+2}^{(r)}
\end{array}\right)=Q_{r+2}\left(\begin{array}{c}
F_{n}^{(r)} \\
F_{n+1}^{(r)} \\
\vdots \\
F_{n+r+1}^{(r)}
\end{array}\right)
$$

where $Q_{r+2}$ is a square matrix

$$
Q_{r+2}=\left(\begin{array}{cccccc}
0 & 1 & 0 & \cdots & 0 & 0 \\
0 & 0 & 1 & \cdots & 0 & 0 \\
\vdots & \vdots & \vdots & \ddots & \vdots & \vdots \\
0 & 0 & 0 & \cdots & 1 & 0 \\
0 & 0 & 0 & \cdots & 0 & 1 \\
q_{1} & q_{2} & q_{3} & \cdots & q_{r+1} & q_{r+2}
\end{array}\right) .
$$

In order to determine elements $q_{1}, \ldots, q_{r+2}$ we use the fact that terms from $-r$ through 0 of the $r$-th generation hyperfibonacci numbers takes values 0 ,

$$
\ldots \pm 1,0,0, \ldots, 0,1, r+1, \ldots
$$

This follows from Lemma 1 since we have

$$
\left(\begin{array}{c}
(n-2)+r \\
r-1
\end{array}\right)=\frac{n(n+1)(n+2) \cdots(n+r-2)}{(r-1) !} .
$$

These expressions are obviously equal to 0 for $n=0,-1, \ldots,-r$.

In particular, when $n=-r+2$ we get

$$
\left(\begin{array}{c}
0 \\
0 \\
\vdots \\
0 \\
1 \\
F_{2}^{(r)}
\end{array}\right)=\left(\begin{array}{cccccc}
0 & 1 & 0 & \cdots & 0 & 0 \\
0 & 0 & 1 & \cdots & 0 & 0 \\
\vdots & \vdots & \vdots & \ddots & \vdots & \vdots \\
0 & 0 & 0 & \cdots & 1 & 0 \\
0 & 0 & 0 & \cdots & 0 & 1 \\
q_{1} & q_{2} & q_{3} & \cdots & q_{r+1} & q_{r+2}
\end{array}\right)\left(\begin{array}{c}
0 \\
0 \\
\vdots \\
0 \\
0 \\
1
\end{array}\right)
$$


meaning that $q_{r+2}=F_{2}^{(r)}$. In the same way we obtain relations for all elements of $Q_{r+2}$,

$$
\begin{aligned}
q_{r+2}= & F_{2}^{(r)} \\
q_{r+1}= & F_{3}^{(r)}-F_{2}^{(r)} q_{r+2} \\
q_{r}= & F_{4}^{(r)}-F_{3}^{(r)} q_{r+2}-F_{2}^{(r)} q_{r+1} \\
& \cdots \\
q_{1}= & F_{r+3}^{(r)}-F_{r+2}^{(r)} q_{r+2}-\cdots-F_{2}^{(r)} q_{2} .
\end{aligned}
$$

This reasoning gives the next Theorem 1.

Theorem 1. For the hypefibonacci sequences we have

$$
A_{r, n}=Q_{r+2}^{n} A_{r, 0} .
$$

Proof. Relation (13) can be written as $A_{r, n}=Q_{r+2} A_{r, n-1}$. Now the statement of theorem follows immediately,

$$
\begin{aligned}
A_{r, n} & =Q_{r+2} A_{r, n-1}=Q_{r+2}^{2} A_{r, n-2} \\
& =Q_{r+2}^{n} A_{r, 0} .
\end{aligned}
$$

Elements $q_{1}, \ldots, q_{r+2}$ can be expressed explicitly. In particular, expressions for $q_{r}, q_{r+1}, q_{r+2}$ are

$$
\begin{aligned}
q_{r+2} & =1+r \\
q_{r+1} & =1-\left(\begin{array}{c}
r+1 \\
2
\end{array}\right) \\
q_{r} & =\frac{r^{3}-7 r}{6} .
\end{aligned}
$$

As an example we calculate hyperfibonacci numbers $F_{3}^{(2)}, F_{4}^{(2)}, \ldots, F_{9}^{(2)}$ of the second generation, collected in the matrix $A_{2,3}$. For the second generation of the hyperfibonacci sequences we have

$$
\begin{aligned}
A_{2,0}= & \left(\begin{array}{cccc}
0 & 1 & 3 & 7 \\
1 & 3 & 7 & 14 \\
3 & 7 & 14 & 26 \\
7 & 14 & 26 & 46
\end{array}\right) \\
Q_{4}= & \left(\begin{array}{cccc}
0 & 1 & 0 & 0 \\
0 & 0 & 1 & 0 \\
0 & 0 & 0 & 1 \\
1 & -1 & -2 & 3
\end{array}\right),
\end{aligned}
$$


according to (5) and (14). Now we determine the matrix $A_{2,3}$ by Theorem 1 .

$$
A_{2,3}=\left(\begin{array}{cccc}
0 & 1 & 0 & 0 \\
0 & 0 & 1 & 0 \\
0 & 0 & 0 & 1 \\
1 & -1 & -2 & 3
\end{array}\right)^{3}\left(\begin{array}{cccc}
0 & 1 & 3 & 7 \\
1 & 3 & 7 & 14 \\
3 & 7 & 14 & 26 \\
7 & 14 & 26 & 46
\end{array}\right)=\left(\begin{array}{cccc}
7 & 14 & 26 & 46 \\
14 & 26 & 46 & 79 \\
26 & 46 & 79 & 133 \\
46 & 79 & 133 & 221
\end{array}\right) .
$$

Note that the eigenvalues of $Q_{4}$ are $\phi, 1,1, \bar{\phi}$, where

$$
\phi=\frac{1+\sqrt{5}}{2}, \bar{\phi}=\frac{1-\sqrt{5}}{2} .
$$

A class of matrices (14) has some further interesting properties. Here we point out that the determinant of such a matrix is -1 . This is demonstrated in the following

Lemma 2. For $r \in \mathbb{N}$ the determinant of a matrix $Q_{r+2}$ takes value -1 ,

$$
\operatorname{det}\left(Q_{r+2}\right)=-1 .
$$

Proof. We prove this statement by means of comparing determinants of matrices $A_{r,-r}$ and $A_{r,-r-1}$,

$$
A_{r,-r}=Q_{r+2} A_{r,-r-1} .
$$

For matrix $A_{r,-r-1}$ we have

$$
\begin{aligned}
\operatorname{det}\left(A_{r,-r-1}\right) & =\operatorname{det}\left(\begin{array}{ccccc}
(-1)^{r} & 0 & 0 & \cdots & 0 \\
0 & 0 & 0 & \cdots & 1 \\
\vdots & \vdots & \vdots & . & \vdots \\
0 & 0 & 1 & \cdots & F_{r-2}^{(r)} \\
0 & 1 & r+1 & \cdots & F_{r-1}^{(r)}
\end{array}\right)_{r \times r} \\
& =(-1)^{r} \operatorname{det}\left(\begin{array}{ccccc}
0 & 0 & \cdots & 0 & 1 \\
0 & 0 & \cdots & 1 & r+1 \\
\vdots & \vdots & \cdots & \vdots & \vdots \\
0 & 1 & \cdots & F_{r-3}^{(r)} & F_{r-2}^{(r)} \\
1 & r+1 & \cdots & F_{r-2}^{(r)} & F_{r-1}^{(r)}
\end{array}\right)_{(r-1) \times(r-1)} \\
& =(-1)^{r+1}(-1)^{\lfloor(r-1) / 2\rfloor+1} \\
& =(-1)^{\lfloor r / 2\rfloor} .
\end{aligned}
$$

On the other hand, $\operatorname{det}\left(A_{r,-r}\right)=(-1)^{\lfloor r / 2\rfloor}$ which proves that

$$
\operatorname{det}\left(A_{r,-r}\right)=-\operatorname{det}\left(A_{r,-r-1}\right) .
$$

Now, the statement of lemma follows immediately by the Binet-Cauchy theorem. 
It is worth mentioning that in [5] authors give some properties of the $k$-generalized Fibonacci $Q$-matrix.

\section{Cassini identity in a matrix form}

\section{Lemma 3.}

$$
\operatorname{det}\left(\begin{array}{ccc}
F_{n}-1 & F_{n+1}-1 & F_{n+2}-1 \\
F_{n+1}-1 & F_{n+2}-1 & F_{n+3}-1 \\
F_{n+2}-1 & F_{n+3}-1 & F_{n+4}-1
\end{array}\right)=(-1)^{n}, n \geq 0 .
$$

Proof. Using the definition of Fibonacci numbers and elementary transformations on rows and columns of determinants we get

$$
\begin{aligned}
& \operatorname{det}\left(\begin{array}{ccc}
F_{n}-1 & F_{n+1}-1 & F_{n+2}-1 \\
F_{n+1}-1 & F_{n+2}-1 & F_{n+3}-1 \\
F_{n+2}-1 & F_{n+3}-1 & F_{n+4}-1
\end{array}\right) \\
= & \operatorname{det}\left(\begin{array}{ccc}
F_{n}-1 & F_{n+1}-1 & F_{n+2}-1 \\
F_{n+1}-1 & F_{n+2}-1 & F_{n+3}-1 \\
F_{n}+F_{n+1}-1 & F_{n+1}+F_{n+2}-1 & F_{n+2}+F_{n+3}-1
\end{array}\right) \\
= & \operatorname{det}\left(\begin{array}{ccc}
F_{n}-1 & F_{n+1}-1 & F_{n+2}-1 \\
F_{n+1}-1 & F_{n+2}-1 & F_{n+3}-1 \\
1 & 1 & 1
\end{array}\right)=\operatorname{det}\left(\begin{array}{ccc}
F_{n}-1 & F_{n+1}-1 & F_{n}+F_{n+1}-1 \\
F_{n+1}-1 & F_{n+2}-1 & F_{n+1}+F_{n+2}-1 \\
1 & 1 & 1
\end{array}\right) \\
= & \operatorname{det}\left(\begin{array}{ccc}
F_{n}-1 & F_{n+1}-1 & 1 \\
F_{n+1}-1 & F_{n+2}-1 & 1 \\
1 & 1 & -1
\end{array}\right)=\operatorname{det}\left(\begin{array}{ccc}
F_{n} & F_{n+1} & 0 \\
F_{n+1} & F_{n+2} & 0 \\
1 & 1 & -1
\end{array}\right) \\
= & -\left(F_{n} F_{n+2}-F_{n+1}^{2}\right)=(-1)^{n}
\end{aligned}
$$

Lemma 4. For the first generation of hyperfibonacci sequences $\left(F_{n}^{(1)}\right)_{n \geq 0}$

$$
\operatorname{det}\left(\begin{array}{lll}
F_{n}^{(1)} & F_{n+1}^{(1)} & F_{n+2}^{(1)} \\
F_{n+1}^{(1)} & F_{n+2}^{(1)} & F_{n+3}^{(1)} \\
F_{n+2}^{(1)} & F_{n+3}^{(1)} & F_{n+4}^{(1)}
\end{array}\right)=(-1)^{n}
$$

Proof. By using relation

$$
F_{n}^{(1)}=F_{n+2}-1
$$

(that immediately follows from the elementary Fibonacci identity $\sum_{k=0}^{n} F_{k}=$ $\left.F_{n+2}-1, n \geq 0\right)$ and Lemma 3 we have

$\operatorname{det}\left(\begin{array}{lll}F_{n}^{(1)} & F_{n+1}^{(1)} & F_{n+2}^{(1)} \\ F_{n+1}^{(1)} & F_{n+2}^{(1)} & F_{n+3}^{(1)} \\ F_{n+2}^{(1)} & F_{n+3}^{(1)} & F_{n+4}^{(1)}\end{array}\right)=\operatorname{det}\left(\begin{array}{lll}F_{n+2}-1 & F_{n+3}-1 & F_{n+4}-1 \\ F_{n+3}-1 & F_{n+4}-1 & F_{n+5}-1 \\ F_{n+4}-1 & F_{n+5}-1 & F_{n+6}-1\end{array}\right)=(-1)^{n}$ 
Theorem 2. For the sequence $\left(F_{k}^{(r)}\right)_{k \geq 0}, r \in \mathbb{N}$ and $n \in \mathbb{Z}$ a determinant of a matrix $A_{r, n}$ takes values \pm 1 ,

$$
\operatorname{det}\left(A_{r, n}\right)=(-1)^{n+\left\lfloor\frac{r+3}{2}\right\rfloor} .
$$

Proof. Using elementary transformations on matrix and Lemma 2 we get

$$
\begin{aligned}
& \operatorname{det}\left(A_{r, 0}\right)=\operatorname{det}\left(\begin{array}{ccccc}
F_{0}^{(r)} & F_{1}^{(r)} & \cdots & F_{r-2}^{(r)} & F_{r-1}^{(r)} \\
F_{1}^{(r)} & F_{2}^{(r)} & \cdots & F_{r-1}^{(r)} & F_{r}^{(r)} \\
\vdots & \vdots & & \vdots & \vdots \\
F_{r-2}^{(r)} & F_{r-1}^{(r)} & \cdots & F_{2 r-4}^{(r)} & F_{2 r-3}^{(r)} \\
F_{r-1}^{(r)} & F_{r}^{(r)} & \cdots & F_{2 r-3}^{(r)} & F_{2 r-2}^{(r)}
\end{array}\right) \\
& =-\operatorname{det}\left(\begin{array}{ccccc}
0 & F_{0}^{(r)} & \cdots & F_{r-3}^{(r)} & F_{r-2}^{(r)} \\
F_{0}^{(r)} & F_{1}^{(r)} & \cdots & F_{r-2}^{(r)} & F_{r-1}^{(r)} \\
\vdots & \vdots & & \vdots & \vdots \\
F_{r-3}^{(r)} & F_{r-2}^{(r)} & \cdots & F_{2 r-5}^{(r)} & F_{2 r-4}^{(r)} \\
F_{r-2}^{(r)} & F_{r-1}^{(r)} & \cdots & F_{2 r-4}^{(r)} & F_{2 r-3}^{(r)}
\end{array}\right) \\
& =(-1)^{r} \operatorname{det}\left(\begin{array}{ccccc}
0 & 0 & \cdots & 0 & 1 \\
0 & 0 & \cdots & 1 & F_{2}^{(r)} \\
\vdots & \vdots & . \cdot & \vdots & \vdots \\
0 & 1 & \cdots & F_{r-2}^{(r)} & F_{r-1}^{(r)} \\
1 & F_{2}^{(r)} & \cdots & F_{r-1}^{(r)} & F_{r}^{(r)}
\end{array}\right) \\
& =(-1)^{r}(-1)^{\left\lfloor\frac{r+2}{2}\right\rfloor} \operatorname{det}\left(\begin{array}{ccccc}
1 & F_{2}^{(r)} & \ldots & F_{r-1}^{(r)} & F_{r}^{(r)} \\
0 & 1 & \cdots & F_{r-2}^{(r)} & F_{r-1}^{(r)} \\
\vdots & \vdots & \ddots & \vdots & \vdots \\
0 & 0 & \cdots & 1 & F_{2}^{(r)} \\
0 & 0 & \cdots & 0 & 1
\end{array}\right) \\
& =(-1)^{\left\lfloor\frac{r+3}{2}\right\rfloor} .
\end{aligned}
$$

According to Theorem 1 we obtain

$$
\begin{aligned}
\operatorname{det}\left(A_{r, n}\right) & =\operatorname{det}\left(Q_{r+2}\right)^{n} \operatorname{det}\left(A_{r, 0}\right)=(-1)^{n} \operatorname{det}\left(A_{r, 0}\right) \\
& =(-1)^{n}(-1)^{\left\lfloor\frac{r+3}{2}\right\rfloor}=(-1)^{n+\left\lfloor\frac{r+3}{2}\right\rfloor},
\end{aligned}
$$

which completes the statement of the theorem.

Let $M=M^{(m, n, r)}$ be a matrix with $M_{i, j}=F_{n+i+j-2}^{(r)}, 1 \leq i, j \leq m$. Theorem 2 can be restated as

$$
\operatorname{det}\left(M^{(n, r, r+2)}\right)=(-1)^{n+\left\lfloor\frac{r+3}{2}\right\rfloor} .
$$


At the end let us show that for $m>r+2$ the following equlity holds:

$$
\operatorname{det}\left(M^{(m, n, r)}\right)=0 \text {. }
$$

The proof of (21) consists of performing elementary transformations on $M^{(m, n, r)}$ leading to a matrix having one column consisting of zeroes.

Take a look at the $i-t h$ row of $M^{(m, n, r)}$ :

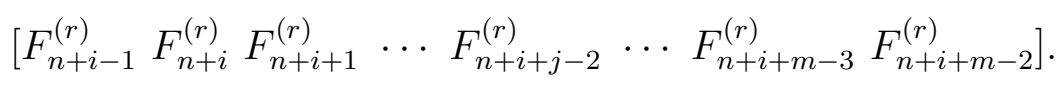

Using (6) and subtracting $j-t h$ element from $(j+1)-s t$ for $j=m-$ $1, m-2, \ldots, 2,1$ (thus simulating subtracting a column $j$ from column $j+1$ in a matrix $M^{(m, n, r)}$ ) we get

$$
\left[\begin{array}{lllllll}
F_{n+i-1}^{(r)} & F_{n+i}^{(r-1)} & F_{n+i+1}^{(r-1)} & \cdots & F_{n+i+j-2}^{(r-1)} & \cdots & F_{n+i+m-3}^{(r-1)} \\
F_{n+i+m-2}^{(r-1)}
\end{array}\right] .
$$

We can repeat the process for $j=m-1, m-2, \ldots, 3,2$ and get

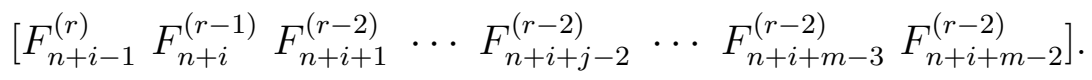

After repeating the process $r-t h$ time (for $j=m-1, m-2, \ldots, r$ ), we get

$$
\left[F_{n+i-1}^{(r)} F_{n+i}^{(r-1)} F_{n+i+1}^{(r-2)} \cdots F_{n+i+r-2}^{(1)} F_{n+i+r-1} \cdots F_{n+i+m-2}\right] .
$$

Since $m>r+2$ we have $n+i+r-1 \leq n+i+m-4$ so the above row contains

$$
\left[\cdots F_{n+i+r-1} F_{n+i+r} F_{n+i+r+1} \cdots\right]
$$

at positions $r-1, r$ and $r+1$. Subtracting first two elements from the third, we get

$$
\left[\cdots F_{n+i+r-1} F_{n+i+r} 0 \cdots\right] .
$$

That way we arrive at a matrix with column consisiting of zeroes whose determinant is therefore zero.

\section{References}

[1] N.N. Cao, F. Z. Zhao, Some Properties of Hyperfibonacci and Hyperlucas Numbers, J. Integer Seq. 13, article 10.8.8 1-11 (2010)

[2] A. Dill, I. Mezö, A symmetric algorithm for hyperharmonic and Fibonacci numbers, Appl. Math. Comput. 206, 942-951 (2008)

[3] R. L. Graham, D. E. Knuth, O. Patashnik, Concrete Mathematics, Addison-Wesley (1994) 
[4] C. Krattenthaler, A. M. Oller-Marcén, A Determinant of Generalized Fibonacci Numbers, J. Combin. Number Theory 5(2), article 2 1-7 (2003)

[5] G.Y. Lee, S.G. Lee, H.G. Shin, On the K-Generalized Fibonacci Matrix $Q_{k}^{*}$, Linear Algebra Appl. 251, 73-88 (1997)

[6] E. Miles, Generalized Fibonacci numbers and related matrices, Amer. Math. Monthly 67, 745-752 (1960)

[7] N. G. Voll, The Cassini Identity and Its Relatives, Fibonacci Quart. 48(3), 197-201 (2010)

[8] S. Vajda, Fibonacci and Lucas numbers, and the golden section, John Wiley Sons, New York (1989)

[9] M. Werman, D. Zeilberger, A Bijective proof of Cassini's Fibonacci identity, Discrete Math. 58, 109 (1986) 